\title{
Correspondence
}

\section{HLA B27, ankylosing spondylitis, and some normal rabbit sera}

SIR, Some time ago we gave an account in this journal of our unsuccessful search for serological cross-reactions between Yersinia enterocolitica and lymphocytes from both HLA B27 normal subjects (B27+ AS-) and HLA B27+ ankylosing spondylitis (AS) patients (B27+ AS+). Our purpose in conducting these experiments had been to see if we could find support for the molecular mimicry hypotheses of AS. ${ }^{1}$

We have continued this search and now wish to report some unexpected results which are in themselves of potential interest and at the same time demonstrate the need for caution in the interpretations of results in experiments concerning bacterial antisera and human lymphocytes.

Our methods for antiserum production and lymphocytotoxicity (LCT) testing were basically the same as those used previously ${ }^{1}$ but with some modifications. We changed to using formalised vaccines for the preparation of rabbit antisera following the report by Geczy et al. ${ }^{2}$ that the putative cross-reacting klebsiella antigen is heat-labile, and for the LCT we used antisera in dilutions as follows: neat, $1+1$, $1+2,1+3,1+4$, and $1+5$, and each of these dilutions with complement dilutions as follows: neat, $1+1,1+2,1+4$. In addition to the $Y$. enterocolitica sera we also made one from Klebsiella pneumoniae (obtained from Dr A. Ebringer, Middlesex Hospital patient 100).

When we used the formalised vaccine prepared antisera we obtained positive results with $Y$. enterocolitica type 9 antiserum even in the standard LCT test described in our previous report, and after these initial positive results we included preimmunisation sera in our tests. As a result of these tests we concluded that the positive results were due to differences in the rabbits we were using, not the change in the method of vaccine production. The results are shown in Table 1 and indicate clearly the preimmunisation serum, that is, apparently normal rabbit serum is capable of causing the cytotoxic effects, and in some cases the kill rate was higher with the preimmunisation serum than that with the postimmunisation one. The fact that the serum contained a high titre of $Y$. enterocolitica type 9 as measured by haemagglutination and gel-diffusion techniques appears to be totally irrelevant. We obtained a few positive results with the $K$. pneumoniae serum, but discontinued the use of it because the kill rate was not as high as it was with the $Y$. enterocolitica. However, those few results indicate that the LCT factor was present in the serum of 2 out of 12 rabbits used in the experiments.

Our results do not invalidate the work of Geczy et al. ${ }^{2}$ concerning the modification of B27+ AS- lymphocytes by klebsiella culture filtrates, so that they become susceptible to lysis by anti-klebsiella sera.

However, it is possible that some of the observations of
Table 1 Percentage kills of lymphocytes by pre-and post-Yersinia enterocolitica type 9 antisera

\begin{tabular}{|c|c|c|c|c|}
\hline & \multicolumn{2}{|c|}{ Lymphocytes } & \multirow{2}{*}{$\begin{array}{l}\text { Preimmunisation } \\
\text { NT }\end{array}$} & \multirow{2}{*}{$\frac{\text { Postimmunisation }}{0 \%}$} \\
\hline 1 & B27+ & $\mathbf{A S}+$ & & \\
\hline 2 & B27+ & $\mathbf{A S}+$ & NT & $40 \%$ \\
\hline 3 & B27+ & AS + & NT & $50 \%$ \\
\hline 4 & B27+ & $\mathrm{AS}+$ & NT & $80 \%$ \\
\hline 5 & B27+ & AS+ & NT & $0 \%$ \\
\hline 6 & B27+ & AS+ & NT & $30 \%$ \\
\hline 7 & B27+ & AS+ & NT & $0 \%$ \\
\hline 8 & B27+ & $\mathbf{A S}+$ & NT & $15 \%$ \\
\hline 9 & B27+ & $\mathbf{A S}+$ & NT & $90 \%$ \\
\hline 10 & B27+ & AS+ & $40 \%$ & $10 \%$ \\
\hline 11 & B27+ & $\mathbf{A S}+$ & $20 \%$ & $15 \%$ \\
\hline 12 & B27+ & AS+ & $60 \%$ & $40 \%$ \\
\hline 13 & B27+ & $\mathbf{A S}+$ & $40 \%$ & $0 \%$ \\
\hline 14 & B27+ & $\mathbf{A S}+$ & $15 \%$ & $10 \%$ \\
\hline 15 & B27+ & $\mathbf{A S}+$ & $40 \%$ & $40 \%$ \\
\hline 16 & B27+ & AS+ & $0 \%$ & $20 \%$ \\
\hline 17 & B27+ & AS+ & $30 \%$ & $0 \%$ \\
\hline 18 & B27+ & $\mathbf{A S}+$ & $20 \%$ & $0 \%$ \\
\hline 19 & B27+ & AS- & $0 \%$ & $0 \%$ \\
\hline 20 & B27+ & AS - & $0 \%$ & $0 \%$ \\
\hline 21 & B27+ & AS- & $0 \%$ & $0 \%$ \\
\hline 22 & $\mathbf{B} 27+$ & AS - & $0 \%$ & $0 \%$ \\
\hline 23 & B27+ & AS - & $0 \%$ & $0 \%$ \\
\hline 24 & B27+ & AS- & $0 \%$ & $0 \%$ \\
\hline 25 & 18 Ran & a controls & $0 \%$ & $0 \%$ \\
\hline
\end{tabular}

Shinebaum et al. ${ }^{3}$ could be explicable in terms of our own results, since no preimmunisation sera were available to them at the time of their investigation ( $R$. Shinebaum, personal communication, 1982). These workers reported that the lymphocytes of one AS patient out of 10 were lysed by several ( 11 definitely, 7 equivocally) klebsiella sera out of a total of 52 sera, and it seems possible that some of these sera contained the factor we have encountered.

In our own experiments varying degrees of killing occurred in 15 out of 18 samples from $\mathrm{B} 27+\mathrm{AS}+$ patients, whereas there was none at all in $6 \mathrm{~B} 27+\mathrm{AS}-$ or in 18 random samples of lymphocytes. This, although difficult to interpret at the moment, is potentially of great interest.

PAULINE MACKINTOSH Tissue Typing Laboratory, Blood Transfusion Service, Birmingham B15 2SG.

PHYLLIS PEASE Department of Medical Microbiology, The Medical School, Birmingham B15 2TJ. 


\section{References}

1 Pease P, England J, Tyler R, Mackintosh P. HLA B27, Yersinia enterocolitica, and ankylosing spondylitis. Ann Rheum Dis 1980; 39: 415-6.

2 Geczy A F, Alexander K, Bashir H V. A factor(s) in Klebsiella culture filtrates specifically modifies an HLA-B27 associated cell-surface component. Nature 1980; 283: 782-4.

3 Shinebaum R, Cooke E M, Siegerstetter J, Wright V. Effect of Klebsiella capsular antisera on lymphocytes from patients with ankylosing spondylitis. J Med Microbiol 1981; 14: 451-6.

\section{Visual analogue scales}

SIR, Although the visual analogue scale (VAS) is widely used in rheumatology, especially for the measurement of pain, its use and interpretation require care. It has recently been suggested ${ }^{1}$ that patients using it are unconsciously influenced by the so-called 'golden section', estimating short below $6.19 \mathrm{~cm}$ along the scale and long above that distance. As the methodology of the study which suggested this was not typical of clinical use of the VAS, we asked 100 patients attending the physiotherapy department in South Tees to estimate their pain on a vertical VAS on arrival at the treatment unit. They were then prepared for treatment, and before therapy was commenced filled in a second VAS without having sight of their first scale. When the results of the first and second measurements were compared, $52 \%$ of the second estimates fell short, $17 \%$ were equal, and $31 \%$ were longer than the first. The results are summarised in Table 1 . When results from patients whose original score was $6 \mathrm{~cm}$ or less were compared with those between $6 \cdot 1 \mathrm{~cm}$ and $10 \mathrm{~cm}$, no significant difference was found between the 2 groups (Table 2 ).

Scott and Huskisson ${ }^{2}$ suggested that patients who are not allowed to see their original score tend to over-estimate at a second attempt. Our findings do not accord with theirs, as $52 \%$ of our patients gave a smaller estimate on their second attempt, only $31 \%$ increasing.

Although we agree with Dixon and Bird ${ }^{1}$ that reproducibility is best near the ends of the scale, we did not find the change in estimate from long to short at $6 \mathrm{~cm}$ which they described. We believe this is due to the fact that their

Table 1 Differences in estimates at different sites on the $V A S$

\begin{tabular}{llllll}
\hline $\begin{array}{l}\text { Distance } \\
(\mathrm{cm}) \text { up } \\
V A S\end{array}$ & \multicolumn{2}{l}{ Second estimate } & & $\begin{array}{l}\text { Mean } \\
\text { difference }\end{array}$ & $\begin{array}{l}\text { Standard } \\
\text { deviation }\end{array}$ \\
\cline { 2 - 5 } & Short & Equal & Long & & \\
\hline $0-1$ & 5 & 3 & 6 & $0 \cdot 2$ & $0 \cdot 254$ \\
$1 \cdot 1-2$ & 4 & 3 & 5 & $0 \cdot 283$ & $0 \cdot 327$ \\
$2 \cdot 1-3$ & 8 & 2 & 4 & $0 \cdot 35$ & $0 \cdot 401$ \\
$3 \cdot 1-4$ & 7 & 3 & 4 & $0 \cdot 478$ & $0 \cdot 544$ \\
$4 \cdot 1-5$ & 7 & 3 & 3 & $0 \cdot 331$ & $0 \cdot 382$ \\
$5 \cdot 1-6$ & 7 & 0 & 1 & $0 \cdot 85$ & $0 \cdot 65$ \\
$6 \cdot 1-7$ & 6 & 1 & 2 & $0 \cdot 478$ & $0 \cdot 421$ \\
$7 \cdot 1-8$ & 3 & 0 & 4 & $0 \cdot 643$ & $0 \cdot 378$ \\
$8 \cdot 1-9$ & 1 & 2 & 2 & $0 \cdot 08$ & $0 \cdot 083$ \\
$9 \cdot 1-10$ & 4 & 0 & 0 & $0 \cdot 175$ & $0 \cdot 096$ \\
\hline
\end{tabular}

Table 2 Behaviour of second estimate for different lengths of first estimate

\begin{tabular}{llcc}
\hline $\begin{array}{l}\text { Position of first } \\
\text { estimate }\end{array}$ & \multicolumn{3}{l}{ Second estimate } \\
\cline { 2 - 4 } & Short & Equal & Long \\
\hline$<6 \mathrm{~cm}$ & 38 & 14 & 23 \\
$6 \cdot 1-10 \mathrm{~cm}$ & 14 & 3 & 8 \\
\hline
\end{tabular}

$\chi^{2}=0 \cdot 0124$, NS

subjects were normal volunteers who were trying to memorise the position of an arbitrary line, whereas we used patients who have their own built-in pain to use as a reference point. It appears, therefore, that the golden section phenomenon which they described is an artefact induced by using normal volunteers rather than a real problem in clinical use of visual analogue scales.

\section{P. DAWES \\ IAN HASLOCK \\ Department of Rheumatology, South Tees Health District, Middlesbrough General Hospital, Ayresome Green Lane, Middlesbrough, Cleveland TS5 5AZ.}

\section{References}

1 Dixon J S, Bird H A. Reproducibility along a $10 \mathrm{~cm}$ vertical visual analogue scale. Ann Rheum Dis 1981; 40: 87-9.

2 Scott J, Huskisson E C. Accuracy of subjective measurements made with or without previous scores: an important source of error in measurements of subjective states. Ann Rheum Dis 1979; 38: 558-9.

SIR, We read the above letter of Dr Dawes and Dr Haslock with interest. Our earlier study ${ }^{1}$ simply sought to draw attention to possible variation in reproducibility along the length of a VAS that might occur if we followed recommendations ${ }^{2}$ to allow subjects access to their previous scores. All workers now seem agreed that this variation occurs, ${ }^{3}$ and our subsequent studies on serial exposure to visual analogue scales also confirm it. ${ }^{4}$

We selected normal volunteers in order to exclude any further variation that might arise as patients attempted to reproduce the modality measured (be it pain, stiffness, or sleep) on a $10 \mathrm{~cm}$ line. To have investigated this would have involved inflicting pain or stiffness on our volunteers. Alternatively we could have used patients but felt that their subjective interpretation of pain (or stiffness) would have added too great a source of error.

We suggested in our paper that errors resulting from the 'golden section' phenomenon are certainly in evidence but are a less likely source of error than a variety of other factors that appertain in the clinic. We are therefore pleased that 